\title{
Flexible Multibody Impact Simulations Using Hierarchically Refined Isogeometric Models
}

\author{
Tobias Rückwald, Alexander Held, Robert Seifried \\ Institute of Mechanics and Ocean Engineering \\ Hamburg University of Technology \\ Eißendorfer Straße 42, 21073 Hamburg, Germany \\ \{tobias.rueckwald, alexander.held, robert.seifried\}@ tuhh.de
}

\begin{abstract}
Detailed impact simulations in flexible multibody systems can be simulated based on reduced isogeometric analysis (IGA) models. A precise simulation of an impact requires a high element resolution in the contact area. Usually, global refinement methods are used, which are easy to implement. However, the literature proposes the use of hierarchical refinement to refine locally. The local refinement generates fewer countable degrees of freedom compared to an equivalent global refinement. Numerous application areas can be found in the literature, such as contact simulations, where the computational effort is reduced by local refinement. This work tests the hierarchical refinement in the context of an impact simulation with the floating frame of reference formulation. In the application example, the impact of two elastic spheres is simulated and compared to an analytic solution. The focus is set on calculation time and accuracy compared to globally refined reference models.
\end{abstract}

Keywords: impact simulation, IGA, floating frame of reference formulation.

\section{INTRODUCTION}

In impact problems within flexible multibody systems, the rigid body motions before and after impact are often large while only small elastic deformations occur during impact. This is the case for relatively stiff materials. Flexible bodies made of steel or aluminum are considered stiff, which allows the application of the floating frame of reference formulation [1] in this work. The use of the floating frame of reference formulation requires global shape functions $\boldsymbol{\Phi}$ of the flexible bodies to model the body flexibility. The global shape functions can be obtained with the finite element method [2] and a reduction method, such as the Craig-Bampton method [3]. When simulating flexible multibody systems and impacts, the bodies are usually meshed with isoparametric elements. A major drawback of isoparametric elements is the discretization of the geometry. Since the calculation of the contact force is based on the geometry of the finite element model, errors can occur. An alternative to isoparametric elements is the IGA, whose use is motivated by two advantages. First, the use of non-uniform rational basis splines (NURBS) as local shape functions of the elements allows an exact representation of the body geometry. Second, high modes of flexible bodies are represented more accurately compared to isoparametric elements [4]. The latter advantage is useful for the floating frame of reference formulation introduced in the later course of this work. These advantages are bought by increased computational costs in the evaluation of the nonlinear local shape functions compared to linear shape functions of isoparametric elements.

An accurate impact simulation, which captures the local deformation, requires a high element resolution in the contact area, as this is where the largest elastic deformations and stresses occur. However, the refinement methods usually used in IGA only allow global refinement. When the number of elements is increased in the contact area, additional elements and control points are created over the entire body, as exemplified on the left hand side in Fig. 1. The control points represent the degrees of freedom in the IGA, thus the global refinement greatly increases the number of equations in the finite element model. One method for local refinement is a hierarchical approach, 


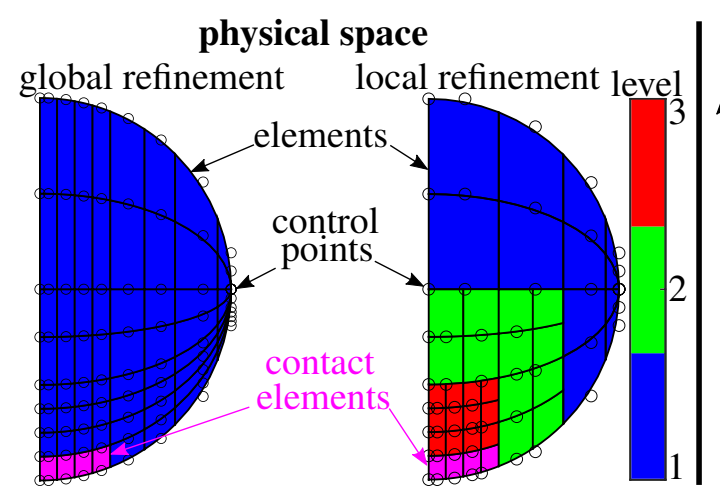

parameter space
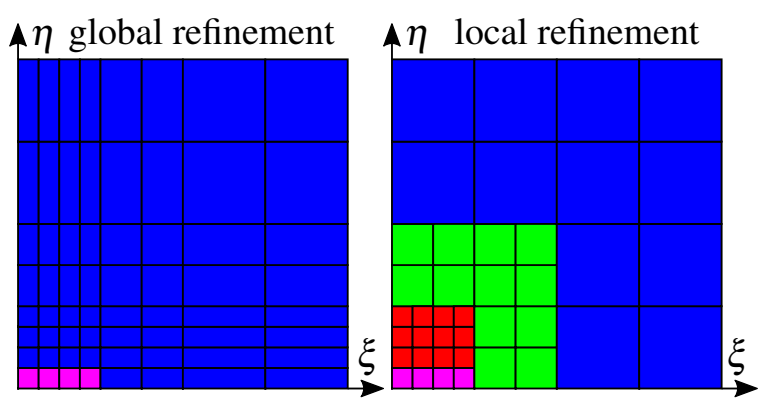

Figure 1: Example of a semicircle that should be refined in the contact area.

where subordinate levels are introduced, as displayed in Fig. 1. The hierarchical refinement is widely used in the literature. It is applied in elementary fluid and structural analysis [5], heat conduction problems [6], topology optimization [7] and contact simulations [8]. The aforementioned literature summarizes that the computational effort can be reduced due to the smaller number of degrees of freedom compared to a global refinement. These examples are mostly from statics, in this work hierarchical refinement is applied in a dynamic problem. The aim of this work is to show whether the computational effort is reduced when simulating the impact of hierarchically refined bodies in the context of the floating frame of reference formulation.

This work is organized in the following way. Initially, the concept of the floating frame of reference formulation is briefly summarized in Section 2. Section 3 introduces the concepts of the IGA, the hierarchical refinement, and the extraction of the global shape functions. The contact algorithm is briefly explained in Section 4. The following Section 5 is a detailed discussion of an application example, and the results are summarized in Section 6.

\section{FLOATING FRAME OF REFERENCE FORMULATION}

When simulating flexible multibody systems, the floating frame of reference formulation is a well-established approach [1]. Large nonlinear rigid body motion of the body frame $K_{R}$ can be described within the inertial frame $K_{I}$. In this work, a Buckens-frame [1] is utilized as floating frame. Provided that the body deformations remain small and linear elastic, they can be described in the body frame $\mathrm{K}_{\mathrm{R}}$. Using the global shape functions $\boldsymbol{\Phi}$ and the $n_{\mathrm{q}}$ elastic coordinates $\boldsymbol{q}_{\mathrm{e}}$, the elastic deformation can be approximated. The equations of motion of a single flexible body are given by

$$
\underbrace{\left[\begin{array}{ccc}
m \mathbf{E} & \mathbf{0} & \boldsymbol{C}_{\mathrm{t}}^{\top} \\
\mathbf{0} & \boldsymbol{I} & \boldsymbol{C}_{\mathrm{r}}^{\top} \\
\boldsymbol{C}_{\mathrm{t}} & \boldsymbol{C}_{\mathrm{r}} & \boldsymbol{M}_{\mathrm{e}}
\end{array}\right]}_{\boldsymbol{M}} \underbrace{\left[\begin{array}{c}
{ }^{\mathrm{R}} \dot{\boldsymbol{v}}_{\mathrm{IR}} \\
\dot{\omega}_{\mathrm{IR}} \\
\ddot{\boldsymbol{q}}_{\mathrm{e}}
\end{array}\right]}_{\dot{z}_{\mathrm{II}}}=\underbrace{\boldsymbol{h}_{\mathrm{p}}+\boldsymbol{h}_{\mathrm{d}}+\boldsymbol{h}_{\mathrm{b}}-\boldsymbol{h}_{\boldsymbol{\omega}}-\boldsymbol{h}_{\mathrm{e}}}_{\boldsymbol{h}_{\mathrm{a}}}
$$

where ${ }^{\mathrm{R}} \boldsymbol{v}_{\mathrm{IR}}$ is the velocity of the rigid body motion from the inertial frame to the reference frame and ${ }^{\mathrm{R}} \omega_{\mathrm{IR}}$ is the angular velocity. In Eq. (1), the mass of the body is denoted by $m$, the translational coupling matrix by $\boldsymbol{C}_{\mathrm{t}}$, the rotational coupling matrix by $\boldsymbol{C}_{\mathrm{r}}$ and the mass moment of inertia by $\boldsymbol{I}$. The right hand side vector $\boldsymbol{h}_{\mathrm{a}}$ is composed of the vector of surface forces $\boldsymbol{h}_{\mathrm{p}}$, the discrete forces $\boldsymbol{h}_{\mathrm{d}}$, the body forces $\boldsymbol{h}_{\mathrm{b}}$, and the inertial forces $\boldsymbol{h}_{\boldsymbol{\omega}}$. The mass matrix $\overline{\boldsymbol{M}}_{\mathrm{e}}$ of a linear elastic body as well as the stiffness $\overline{\boldsymbol{K}}_{\mathrm{e}}$ and damping matrix $\overline{\boldsymbol{D}}_{\mathrm{e}}$ needed to compute the inner forces $\boldsymbol{h}_{\mathrm{e}}$ are introduced in a later section. Contact forces are considered in the discrete forces $\boldsymbol{h}_{\mathrm{d}}$. The standard input data (SID), a well-known standard in providing elastic data in the context of the floating frame of reference formulation [1], is used to assemble the equations of motion (1). For the formulation of the equations of motion and the transient analysis, the MATLAB toolbox DYNMANTO [9] is 
used. The resulting equations of motion are solved by the ode15s MATLAB solver with numerical differentiation formulas (NDF).

\section{GLOBAL SHAPE FUNCTIONS FROM IGA}

Determining the global shape functions $\boldsymbol{\Phi}$ is a key issue in using the floating frame of reference formulation. A general way to determine the global shape functions is to generate a finite element model of the flexible body and then identify the global shape functions from the finite element model using model reduction techniques. This section briefly presents the idea of the IGA and the procedure to obtain the global shape functions from an IGA model. A more detailed introduction to the IGA can be found in [4]. Additionally, the concept of hierarchical refinement and a methodology to locally refine isogeometric models are introduced.

\subsection{Basis Splines}

The IGA consists of three spaces: The physical space, the parameter space, and the index space. The first two spaces are essential for understanding the IGA. The shape functions of isogeometric elements are defined in the parameter space, which can be seen for a 2D example on the left hand side in Fig. 2. The parameter space is divided into elements and spanned by the knot vectors $\boldsymbol{\Xi}=\left[\begin{array}{llll}\xi_{1} & \xi_{2} & \ldots & \xi_{\mathrm{n}+\mathrm{p}+1}\end{array}\right]$ and $\mathcal{H}=\left[\begin{array}{llll}\eta_{1} & \eta_{2} & \ldots & \eta_{\mathrm{m}+\mathrm{q}+1}\end{array}\right]$.

Thereby, $p$ and $q$ are the order of the basis functions and $n$ and $m$ are the numbers of the basis functions $N_{i, \mathrm{p}}$ and $M_{j, \mathrm{q}}$. The order of the basis functions can be subsequently increased with the algorithm in [10]. In IGA, the local shape functions are based on B-splines, which can be computed recursively with the Cox-de Boor algorithm [4]. In $\xi$-direction, the B-splines are computed as

$$
\begin{aligned}
& p=0: \quad N_{i, 0}(\xi)= \begin{cases}1 & \text { if } \xi_{i} \leq \xi<\xi_{i+1} \\
0 & \text { otherwise. }\end{cases} \\
& p>1: \quad N_{i, \mathrm{p}}(\xi)=\frac{\xi-\xi_{i}}{\xi_{i+\mathrm{p}}-\xi_{i}} N_{i, \mathrm{p}-1}(\xi)+\frac{\xi_{i+\mathrm{p}+1}-\xi}{\xi_{i+\mathrm{p}+1}-\xi_{i+1}} N_{i+1, \mathrm{p}-1}(\xi) .
\end{aligned}
$$

The calculation rule given by Eq. (2) and Eq. (3) is identical in $\eta$-direction. In practice, recursive functions are numerically inefficient. Therefore, a non-recursive algorithm suggested in [10] is used.

\subsection{Non-Uniform Rational Basis Splines}

Besides the parameter space there is the physical space, which can be seen on the right hand side in Fig. 2. In the physical space, the control points $\boldsymbol{P}_{i, j}$ are defined, which are arranged by the control net. The task of the control points is to span the geometry in the physical space. The dimension

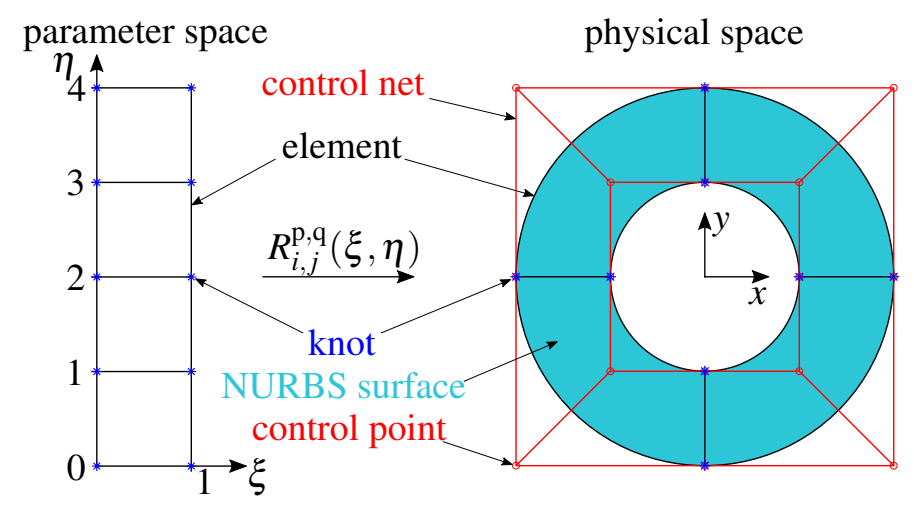

Figure 2: Parameter space and physical space in the IGA. 
of the control points corresponds to the numbers of B-splines $n$ and $m$ in the parameter space. In addition to the physical position, each control point has a weight $w_{i, j}$. The transformation from the parameter space into the physical space requires the NURBS basis $R_{i, j}^{\mathrm{p}, \mathrm{q}}$ given by

$$
R_{i, j}^{\mathrm{p}, \mathrm{q}}(\xi, \eta)=\frac{N_{i, \mathrm{p}}(\xi) M_{j, \mathrm{q}}(\eta) w_{i, j}}{\sum_{\hat{i}=1}^{\mathrm{n}} \sum_{\hat{j}=1}^{\mathrm{m}} N_{\hat{i}, \mathrm{p}}(\xi) M_{\hat{j}, \mathrm{q}}(\eta) w_{\hat{i}, \hat{j}}}
$$

The NURBS basis $R_{i, j}^{\mathrm{p}, \mathrm{q}}$ and the control points $\boldsymbol{P}_{i, j}$ then lead to the NURBS surface

$$
\boldsymbol{S}=\sum_{i=1}^{\mathrm{n}} \sum_{j=1}^{\mathrm{m}} R_{i, j}^{\mathrm{p}, \mathrm{q}}(\xi, \eta) \boldsymbol{P}_{i, j}
$$

in the physical space. The degrees of freedom in the IGA correspond to the displacements of the control points

$$
\boldsymbol{u}_{i, j}=\boldsymbol{P}_{i, j}-\boldsymbol{P}_{i, j}^{0},
$$

whereby $\boldsymbol{P}_{i, j}^{0}$ represents the position of the control points in the undeformed and $\boldsymbol{P}_{i, j}$ in the deformed configuration. The deformation $\boldsymbol{d}$ of the NURBS surface can be written in matrix-vector notation as

$$
\boldsymbol{d}=\underbrace{\left[\begin{array}{cccccc}
R_{1,1}^{\mathrm{p}, \mathrm{q}} & 0 & R_{1,2}^{\mathrm{p}, \mathrm{q}} & 0 & \ldots & 0 \\
0 & R_{1,1}^{\mathrm{p}, \mathrm{q}} & 0 & R_{1,2}^{\mathrm{p}, \mathrm{q}} & \ldots & R_{\mathrm{p}+1, \mathrm{q}+1}^{\mathrm{p}, \mathrm{q}}
\end{array}\right]}_{\boldsymbol{N}} \underbrace{\left[\begin{array}{c}
u_{1,1, \mathrm{x}} \\
u_{1,1, \mathrm{y}} \\
u_{1,2, \mathrm{x}} \\
u_{1,2, \mathrm{y}} \\
\vdots \\
u_{\mathrm{p}+1, \mathrm{q}+1, \mathrm{y}}
\end{array}\right]}_{\boldsymbol{u}},
$$

where the basis functions of the corresponding element are summarized in matrix $N$.

\subsection{Hierarchical Refinement}

The concept of the hierarchical refinement relies on the property of B-splines to be represented by a linear combination of finer B-splines defined on smaller knot-intervals. With the calculation rule

$$
a_{j}=2^{-p}\left(\begin{array}{c}
p+1 \\
j
\end{array}\right)=2^{-p} \frac{(p+1) !}{j !(p+1-j) !}
$$

linear coefficients can be determined to represent a B-spline in a higher level with B-splines of lower level. It should be noted that Eq. (8) is only valid for uniform knot vectors. As an example, a quadratic B-spline with the high-level knot vector

$$
\boldsymbol{\Xi}^{1}=\left[\begin{array}{llll}
0 & 1 & 2 & 3
\end{array}\right]
$$

should be represented by a number of lower level B-splines with the corresponding low-level knot vector

$$
\Xi^{2}=\left[\begin{array}{lllllll}
0 & \underline{0.5} & 1 & \underline{1.5} & 2 & \underline{2.5} & 3
\end{array}\right] .
$$

The inserted knots in Eq. (10) are underlined. By applying Eq. (8) the concept of the hierarchical refinement can be visualized in Fig. 3. The procedure, which is implemented and used in this work, is briefly summarized in the following. For a detailed introduction to the hierarchical refinement, see [5].

Initially, the parameter space is divided into the different hierarchy levels. Recapturing the motivation example in Fig. 1, the corresponding parameter space is displayed on the right hand side. This division is made up by intervals in knot coordinates. The concept of hierarchical refinement is that a finer mesh resolution can be defined section by section. Therefore, the levels are initialized using 


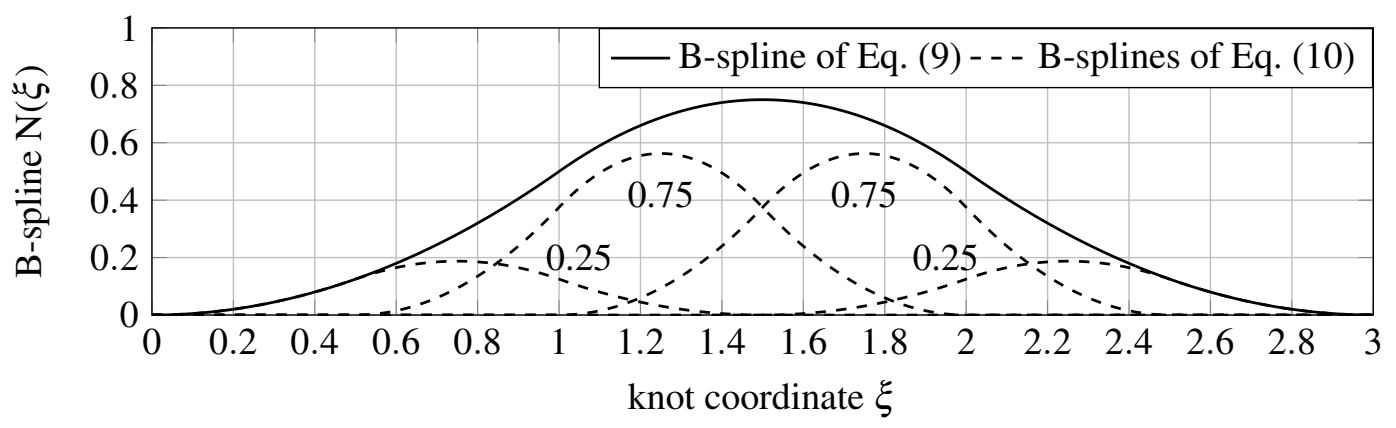

Figure 3: Concept of the hierarchical refinement in the IGA.

the global knot insertion algorithm described in [4]. With this algorithm, the position of the control points and their weights can be determined for the finer mesh. The number of hierarchy levels is denoted as $n_{\mathrm{lvl}}$.

In hierarchical refinement, B-splines of higher level are based on B-splines of lower level. For the construction of higher level B-splines, the linear combination coefficient matrix $\boldsymbol{A}$ is determined by solving

$$
N_{i, \mathrm{p}}^{\ell}=\boldsymbol{A}_{i, j}^{\ell} N_{j, \mathrm{p}}^{\ell+1} \quad \ell=1 \ldots n_{\mathrm{lvl}}-1
$$

between all $n_{\mathrm{lvl}}$ hierarchy levels. A detailed description of this procedure can be found in [11].

In the next step, the elements are defined with the knot vectors of the different levels and the intervals, which defined the hierarchy level with respect to the knot coordinates. The previous step allows certain B-splines to be identified as inactive. Thus, the associated control points of each element can be determined. Thereby, the control points can be located in different hierarchy levels. The control points that are not part of an element are identified as inactive.

The hierarchical B-splines of the example introduced at the beginning in Fig. 1 are depicted in Fig. 4. It can be seen that B-splines of different hierarchy levels can be active, e.g. at the knot coordinate $\xi=0.2$ in Fig. 1. This overlap becomes relevant when calculating the NURBS basis from Eq. (4). The B-splines of the different dimensions of the parameter space need to be multiplied out. These intersections of the B-splines lead to the fact that for the computation of the NURBS any level can interact with any level of the other dimensions in the parameter space. The more hierarchical levels that are created, the more combinations of possible intersections of the B-splines can occur. This may increase the calculation time of the hierarchical NURBS basis compared to a globally refined model. In addition to the more complex calculation of the NURBS, the B-splines from the different hierarchy levels must be constructed, which takes additional computation time.

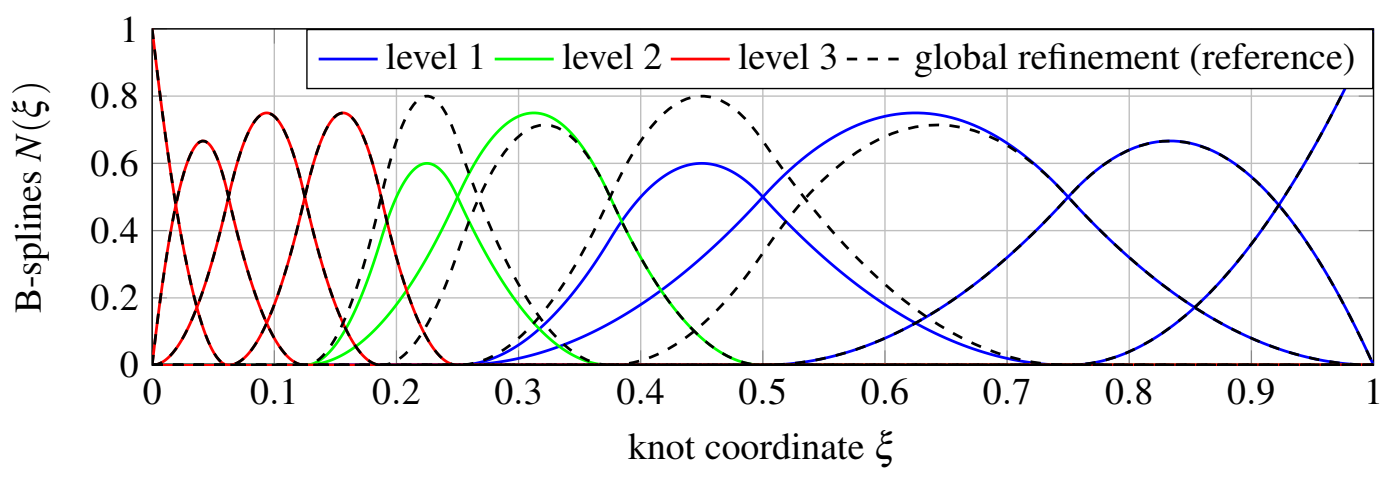

Figure 4: Concept of the hierarchical refinement in the IGA. 


\subsection{IGA Equations of Motion}

As with the floating frame of reference formulation, it is assumed that only small elastic deformations occur in a relatively stiff material. Therefore, linear elasticity is assumed and the weak Galerkin method is applied as for isoparametric elements [2]. The local mass and stiffness matrix of an element is therefore given by

$$
\begin{array}{rlrl}
\boldsymbol{K}_{\mathrm{e}, j} & =\int_{\Omega_{\mathrm{e}, j}} \boldsymbol{B}^{\top} \boldsymbol{C} \boldsymbol{B} \mathrm{d} \Omega_{\mathrm{e}, j} & j=1 \ldots n_{\mathrm{e}}, \\
\boldsymbol{M}_{\mathrm{e}, j}=\rho \int_{\Omega_{\mathrm{e}, j}} \boldsymbol{N}^{\top} \boldsymbol{N} \mathrm{d} \Omega_{\mathrm{e}, j} & j=1 \ldots n_{\mathrm{e}},
\end{array}
$$

where $C$ is the material elasticity matrix and $n_{\mathrm{e}}$ the number of elements. The strain displacement matrix $B$ is obtained by differentiating the NURBS basis $R_{i, j}^{\mathrm{p}, \mathrm{q}}$ and using the Jacobian transformation [2]. The integration over each element $\Omega_{\mathrm{e}, j}$ is performed by Gauss quadrature in the parameter space. The basis functions $\boldsymbol{N}$ and the strain displacement matrix $\boldsymbol{B}$ are evaluated at each Gauss point. As stated in [4], the same Gauss rule for a polynomial of the order $p$ can be applied to a $p$-th order B-spline. Therefore, the Gauss order $p+1$ is chosen in $\xi$-direction and $q+1$ in $\eta$ direction. The global system matrices $\boldsymbol{K}_{\mathrm{e}}$ and $\boldsymbol{M}_{\mathrm{e}}$ are assembled from the corresponding element matrices $\boldsymbol{K}_{\mathrm{e}, j}$ and $\boldsymbol{M}_{\mathrm{e}, j}$. The equations of motion of the complete finite element model are then given by

$$
\boldsymbol{M}_{\mathrm{e}} \ddot{\boldsymbol{u}}_{\mathrm{e}}+\boldsymbol{K}_{\mathrm{e}} \boldsymbol{u}_{\mathrm{e}}=\boldsymbol{f}_{\mathrm{e}},
$$

where the displacements of the control points are represented by $\boldsymbol{u}_{\mathrm{e}}$ and the external forces by $\boldsymbol{f}_{\mathrm{e}}$.

\subsection{Model Order Reduction}

For the incorporation of the isogeometric model into the flexible multibody simulation, the global shape functions $\Phi$ are required. The global shape functions $\Phi$ for Eq. (1) can be determined from the linear system equations (14) with a model order reduction method. The straightforward approach for reducing the equations of motion (14) is modal reduction. However, as shown in [12], modal reduction leads to inaccurate results in case of impact problems, since the local deformation in the contact region is not included in the reduced model. Alternatively, the Craig-Bampton method [3] is used, which has already been successfully applied to impact problems with isoparametric elements [12]. The key idea of the Craig-Bampton method is to combine fixed-interface normal modes and constraint modes. The normal modes represent the overall flexibility and the constraint modes allow a relatively accurate representation of the deformation in a specific area of the flexible body, e.g. the contact area. For the constraint modes, predefined interface control points on the exterior surface can be selected. The procedure results in the global shape functions $\Phi$, which are normalized to the mass matrix. The reduced mass and stiffness matrix are then given by

$$
\overline{\boldsymbol{M}}_{\mathrm{e}}=\boldsymbol{\Phi}^{\top} \boldsymbol{M}_{\mathrm{e}} \boldsymbol{\Phi}=\mathbf{E} \quad \text { and } \quad \overline{\boldsymbol{K}}_{\mathrm{e}}=\boldsymbol{\Phi}^{\top} \boldsymbol{K}_{\mathrm{e}} \boldsymbol{\Phi}=\operatorname{diag}\left(\omega_{i}^{2}\right)
$$

respectively, where $\mathbf{E}$ is the identity matrix and $\omega_{i}$ are the natural frequencies. Since the normal modes tend to be low and the constrained modes high, the equations of motion (1) become numerically stiff. Damping the high modes with the method of [13] and the parameters of [14] increases the numerical performance.

\section{CONTACT HANDLING IN IGA}

There exist several methods for discretizing the contact of two isogeometric bodies. Two frequently used types of methods are the integral description of the contact and the node-to-segment methods. In the integral description of the contact, Gauss points of preselected elements are checked [14]. The number of evaluation points depends on the number of elements in the contact area and on the order of the B-splines due to the Gauss integral. For node-to-segment methods, the number of evaluation 
points depends only on the number of elements in the contact area. Since node-to-segment methods require fewer points to be checked for contact and the accuracy is still comparable to that of an integral description [15], a node-to-segment method is chosen for this work.

For node-to-segment methods, different collocation methods, e.g. Botella points can be used [15]. The following section briefly summarizes the contact algorithm described in [14]. This collocation method is combined with the penalty method for contact treatment. The corresponding penalty factor $c_{\mathrm{p}}$ is chosen heuristically. Thereby, the penalty factor should be chosen large enough such that the results become independent of the chosen parameter [16]. When the penalty factor is increased beyond its converging value, the differential equation (1) becomes stiffer and the simulation time is unnecessarily increased or the simulation might even terminate unsuccessfully.

At each time step, the individual bodies must be checked for contact. To this end, the position of the deformed control points $\boldsymbol{P}_{i, j}$ is determined. First, the control points displacements are recovered

$$
\boldsymbol{u}_{\mathrm{e}}=\boldsymbol{\Phi} \boldsymbol{q}_{\mathrm{e}}
$$

with the global shape functions $\Phi$ and the elastic coordinates $\boldsymbol{q}_{\mathrm{e}}$. After that, the position of the control points is computed from Eq. (6). In case of contact between two bodies, one body is defined as the contact body and the other as the target body, as depicted in Fig. 5. The Botella points, which are located on the outer surface of the contact body and in the contact area, are tested for contact with the exterior surface of the target body. The contact check is achieved by solving

$$
\left(\frac{\partial x_{\mathrm{T}}(\xi)}{\partial \xi}\right)^{\top}\left(x_{\mathrm{C}}-x_{\mathrm{T}}(\xi)\right)=0
$$

with the Newton-Raphson method for the respective knot coordinate, e.g. $\xi$. This knot coordinate $\xi$ corresponds to the target point $\boldsymbol{x}_{\mathrm{T}}(\xi)$, which is closest to the current Bottela point $\boldsymbol{x}_{\mathrm{C}}$ of the contact body. The distance $g_{\mathrm{n}}$ between the contact and target point is determined by

$$
g_{\mathrm{n}}=\boldsymbol{n}^{\top}\left(\boldsymbol{x}_{\mathrm{C}}-\boldsymbol{x}_{\mathrm{T}}(\xi)\right),
$$

where the normal vector $\boldsymbol{n}$ is orthogonal to the surface of the target body. If the normal gap $g_{n}$ is greater than zero, there is no contact. Otherwise, the contact force for the current sampling point is determined by

$$
\boldsymbol{f}_{\mathrm{c}}=c_{\mathrm{p}} g_{\mathrm{n}} \boldsymbol{N}^{\top} \boldsymbol{n} \hat{\omega}_{i},
$$

where $c_{\mathrm{p}}$ is the penalty factor, $N$ the local shape functions and $\hat{\omega}_{i}$ the collocation weight of the current collocation point. See [15] for the derivation of the collocation weight. To eliminate the distinction between contact and target body, they are switched and the contact force is averaged. After the contact search, the discrete forces can be calculated with

$$
\boldsymbol{h}_{\mathrm{d}}=\sum_{i=1}^{\mathrm{n}} \sum_{j=1}^{\mathrm{m}}\left[\begin{array}{c}
\mathbf{E} \\
\tilde{\boldsymbol{P}}_{i, j} \\
\boldsymbol{\Phi}_{i, j}^{\top}
\end{array}\right] \boldsymbol{f}_{\mathrm{c}, i, j}
$$

and inserted into Eq. (1). The contact algorithm described above is implemented in MATLAB. However, for the sake of computational efficiency, the algorithm is compiled into C-code using the MATLAB-MEX toolbox. The contact search can be parallelized with respect to the contact evaluation points.

\section{APPLICATION EXAMPLE: IMPACT OF TWO SPHERES}

The aim of this application example is to compare globally and locally refined isogeometric models in the context of an impact simulation in a flexible multibody system. It is investigated whether a locally refined model has the same accuracy as an equivalent globally refined model and how locally and globally refined models differ in computation time. 
In this application example, the impact of two spheres is simulated. This simple example is wellsuited as a benchmark because an analytical solution exists [17]. For the sake of simplicity, the spheres are identical and, therefore, the problem is symmetric. Despite the symmetry, both spheres are modeled. However, the axisymmetric property of a sphere is exploited by a semicircle. The simulation setup is visualized in Fig. 6 . The radii of the spheres are $r=1 \mathrm{~cm}$ and the selected

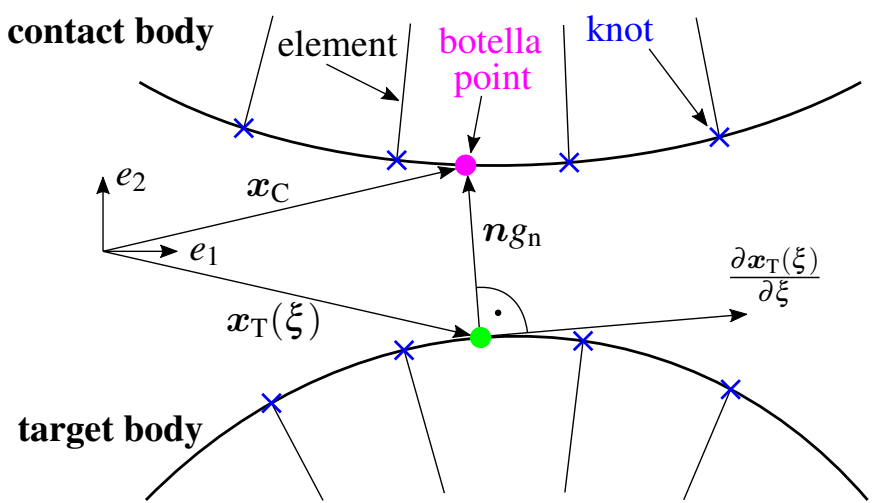

Figure 5: Contact detection between two bodies.

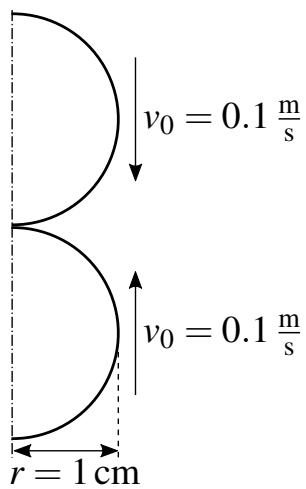

Figure 6: Impact of two spheres.

material is steel. Therefore, the Young's modulus is chosen as $E=2.11 \times 10^{11} \mathrm{~Pa}$, the density as $\rho=7850 \frac{\mathrm{kg}}{\mathrm{m}^{3}}$ and the Poisson's ratio as $v=0.3$. Both spheres have an initial velocity of $v_{0}=0.1 \frac{\mathrm{m}}{\mathrm{s}}$ and gravity is ignored. For the initialization of the geometry the knot vectors of the semicircle are defined as $\boldsymbol{\Xi}=\left[\begin{array}{llllll}0 & 0 & 0 & 1 & 1 & 1\end{array}\right]$ and $\mathcal{H}=\left[\begin{array}{llll}0 & 0 & 1 & 1\end{array}\right]$. The initial order of the B-splines is given by $p=2$ and $q=1$. The control points and weights in the physical space are

$$
\boldsymbol{P}_{i, j, 1}^{0}=\left[\begin{array}{cc}
0 & 0 \\
r & r \\
r & r
\end{array}\right], \quad \boldsymbol{P}_{i, j, 2}^{0}=\left[\begin{array}{cc}
-r & r \\
-r & r \\
0 & 0
\end{array}\right], \quad \text { and } \quad \boldsymbol{w}_{i, j}=\left[\begin{array}{cc}
1 & 1 \\
\frac{1}{\sqrt{2}} & \frac{1}{\sqrt{2}} \\
1 & 1
\end{array}\right] .
$$

After defining the geometry, the model is globally refined to represent the overall elastic deformation better. In this, the order of the B-splines is increased by two resulting in the order $p=2+2=4$ and $q=1+2=3$. Adding 15 knots in $\xi$-and 24 knots in $\eta$-direction increases the number of elements from one to 400 . This refined model serves as a starting point for various benchmark models. It is worth noting, that all tested models have an element edge length of $10 \mu \mathrm{m}$ in the contact area. The models are reduced with the Craig-Bampton method, using ten normal modes, and the exterior control points in the contact area are used for the constrained modes.

Four locally refined models are compared for the following studies. The number of hierarchy levels $n_{\mathrm{lvl}}$ is varied between three and six. Each of these four locally refined models has an equivalent globally refined reference model. The equivalence is that the knot vectors of the globally refined models are identical to the knot vectors in the lowest level of the locally refined models. The investigated models are listed in Table 1 and visualized in Fig. 7. The number of interface control points is nearly identical for all models. Accordingly, the number of elastic coordinates $n_{\mathrm{q}}$ after the model reduction is almost identical regardless of whether the model was globally or locally refined. In addition, it should be emphasized that in this example, the number of degrees of freedom $n_{\text {dof }}$ decreases as the number of hierarchy levels $n_{\mathrm{lvl}}$ increases. The dimensions on the left side of the four IGA models in Fig. 7a-7d represent the height of the lowest hierarchy level, which becomes relevant in the further course of the analysis. The model with five hierarchy levels in Fig. 7c represents the most uniform distribution of elements. Since the goal in this application example is to create an element length of $10 \mu \mathrm{m}$ in the contact area, models with more than six hierarchy levels are not necessary, as shown in Fig. 7d.

The impacts in this work are simulated with a penalty method. As mentioned before, the penalty factor $c_{\mathrm{p}}$ is increased until the results become independent of the penalty factor. In Fig. 8, the 
Table 1: Globally and locally refined benchmark models of an axisymmetric sphere.

\begin{tabular}{c||c|c|c|c} 
locally refined & $n_{\mathrm{lvl}}=3$ & $n_{\mathrm{lvl}}=4$ & $n_{\mathrm{lvl}}=5$ & $n_{\mathrm{lvl}}=6$ \\
\hline \hline $\begin{array}{c}\text { degrees of freedom } n_{\text {dofs }} \\
\text { number of elastic coordinates } n_{\mathrm{q}} \\
\text { globally refined }\end{array}$ & 3856 & 2884 & 2728 & 1708 \\
\hline $\begin{array}{c}\text { degrees of freedom } n_{\text {dofs }} \\
\text { number of elastic coordinates } n_{\mathrm{q}}\end{array}$ & 9216 & $10+80$ & $10+84$ & $10+82$ \\
\hline \hline & $10+82$ & $10+80$ & $10+80$ & $10+78$
\end{tabular}

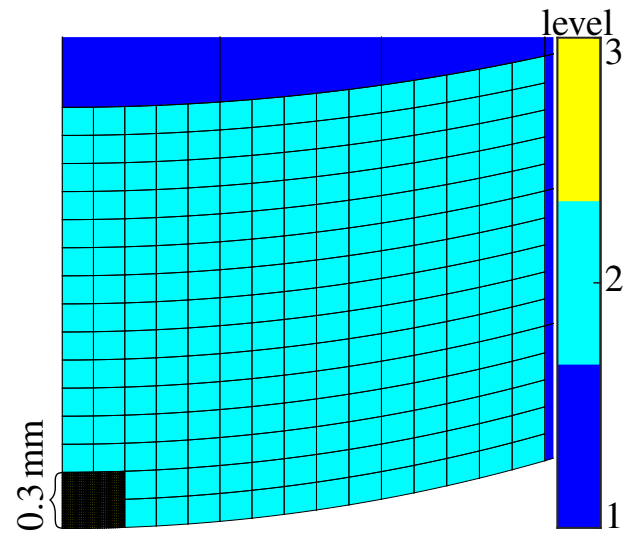

(a) $n_{\mathrm{lvl}}=3$.

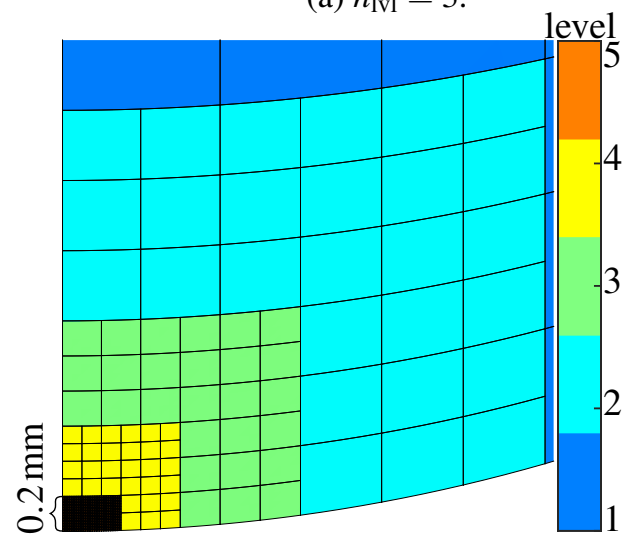

(c) $n_{\mathrm{lvl}}=5$.

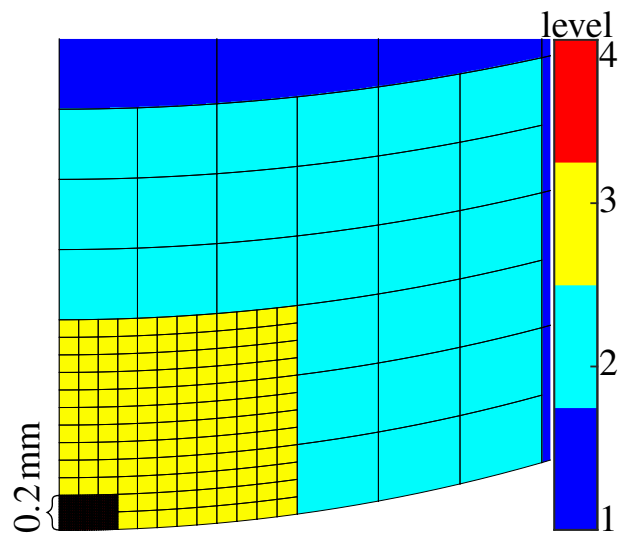

(b) $n_{\mathrm{lvl}}=4$

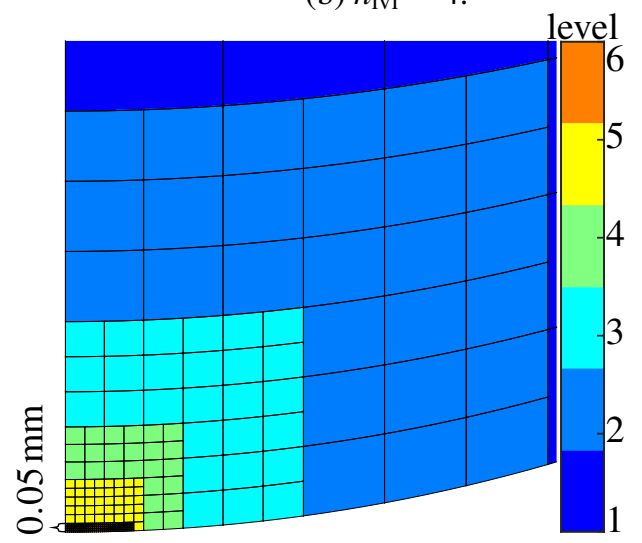

(d) $n_{\mathrm{lvl}}=6$.

Figure 7: Detail plot of contact area of the hierarchical models of the axisymmetric sphere.

maximum relative error of the contact force for the analytic solution [17] is visualized. Two observations can be made from Fig. 8. First, it is observed in Fig. 8a that the penalty factor is independent of the number of hierarchy levels. Second, a globally and locally refined model behaves almost identically here, as seen in Fig. 8b. Figure 8 shows that for the penalty factors $c_{\mathrm{p}}$ greater than $5 \times 10^{18} \mathrm{~N} / \mathrm{m}$ the error of the contact force remains constant at approximately $2 \%$. Therefore, this factor is used for the following simulations. It is noted that the calculation time also increases with increasing penalty factor, since the equations of motion to be solved become stiffer.

Next, the influence of the number of hierarchy levels $n_{\mathrm{lvl}}$ on the computation time of the impact simulation shown in Fig. 9 is discussed. Although the contact search can be parallelized with respect to the evaluation points, sequentially executed simulations are more independent of the architecture of the computer, the MATLAB-MEX implementation of parallel for-loops, the code quality, and the used operating system WINDOWS. Therefore, the sequential computation allows a better relative comparison of the individual models. The processor used is the Intel W-2295 model 


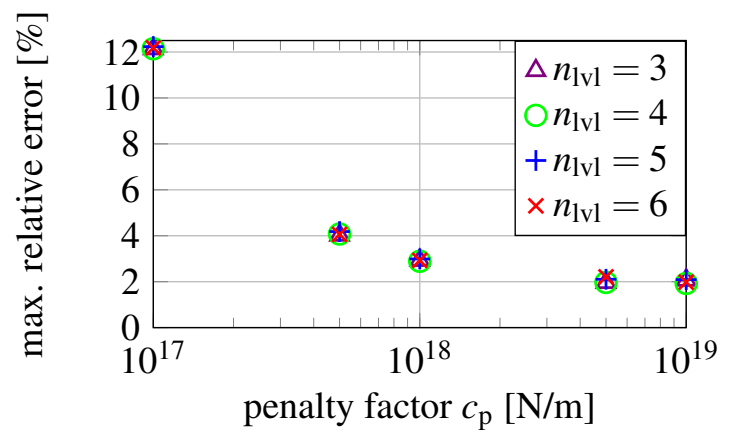

(a) All locally refined models.

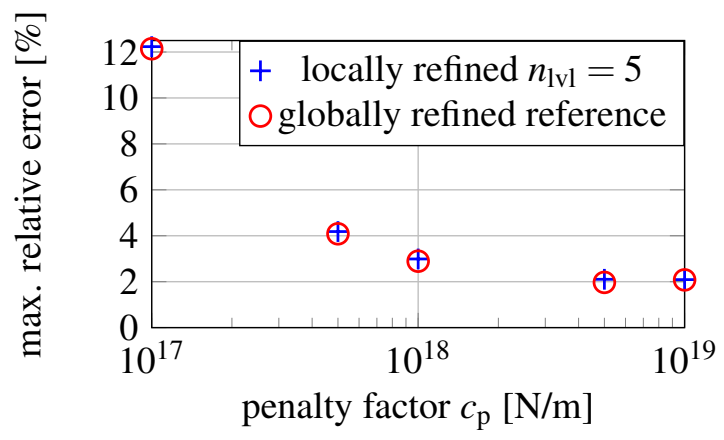

(b) Local $n_{\mathrm{lvl}}=5$ model and its global reference.

Figure 8: Contact force error with respect to the analytic solution by Hertz [17].

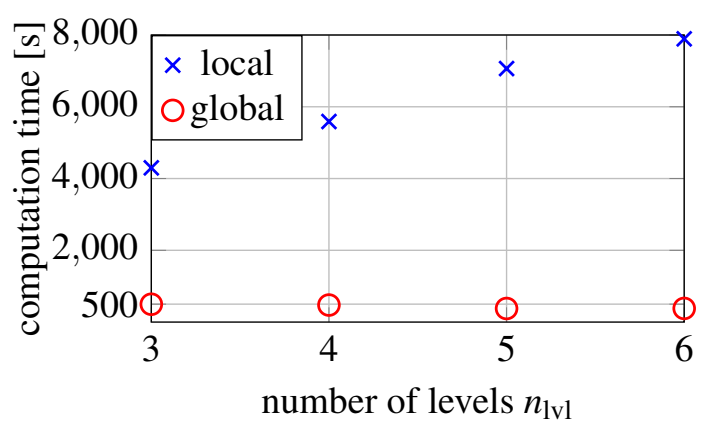

(a) Sequential computing.

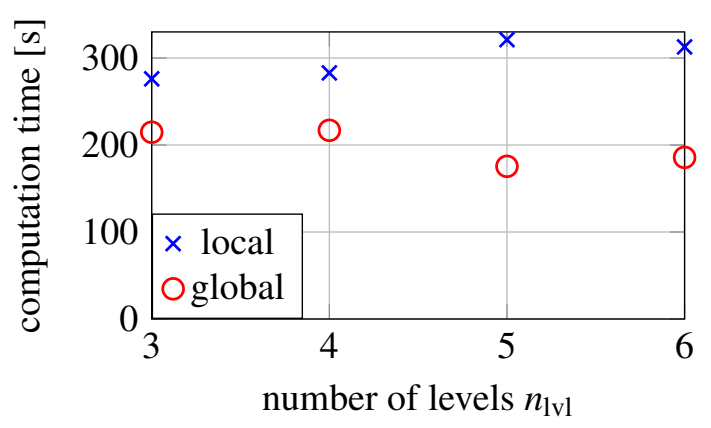

(b) Parallelized computing.

Figure 9: Comparison of computation times.

with 18 cores. It should be noted that the following qualitative observations may depend on the particular application example.

Observing the computation time of the sequential simulation in Fig. 9a, it can be seen that the computation time increases, in this case linearly, as the number of hierarchy levels increases. In contrast, the computation time of the globally refined reference models decreases. The latter observation can be attributed to the fact that the number of degrees of freedom $n_{\text {dof }}$ decreases, see Table 1. Since the globally refined models have only one level in the parameter space, it is likely that the number of degrees of freedom $n_{\text {dof }}$ directly influences the computation time. If the number of degrees of freedom $n_{\text {dof }}$ is smaller, the matrix of global shape functions $\Phi$ is also smaller, and the calculation of Eq. (16) and Eq. (20) is faster.

Although, the degrees of freedom of the locally refined models decrease as the number of hierarchy levels increases, the aforementioned effect does not seem to be dominant here. In the literature $[5,6$, $7,8]$, the degrees of freedom saved by local refinement are listed as an advantage. This advantage does not apply in this work since the models are reduced with the Craig-Bampton method and the reduction of the number of elastic coordinates $n_{\mathrm{q}}$ is almost identical regardless of the type of refinement, see Table 1. In applying the floating frame of reference formulation, the reduced mass and stiffness matrix are used for calculation. The full mass matrix does not have to be inverted in each time step. Only the global shape functions $\boldsymbol{\Phi}$ have to be transposed in Eq. (20) for the contact algorithm, which is much more time-saving than a matrix inversion. Since the number and position of contact evaluation points are also identical for the respective globally and locally refined models, the evaluation of the NURBS in the course of the contact evaluation remains the last possible cause for the difference in computation time. The additional computational effort in computing hierarchical NURBS described in Section 3.3 is responsible for the higher computational time in Fig. 9a. 
In the parallel computation in Fig. 9b, the globally refined models are likewise faster. However, the difference between the globally and locally refined models is smaller compared to the sequential computation. In this case, the locally refined models seem to benefit more from the parallel computation than the globally refined models, whereby the MATLAB-MEX implementation could be responsible for this.

In the last analysis of this application example, the von Mises stresses are observed when they are maximum. The time of maximum occurrence of the von Mises stresses is when the contact force is maximum [17]. With the analytical solution by Hertz [17], the von Mises stresses along the symmetry axis of the sphere are displayed in Fig. 10. Initially, it can be observed that all locally

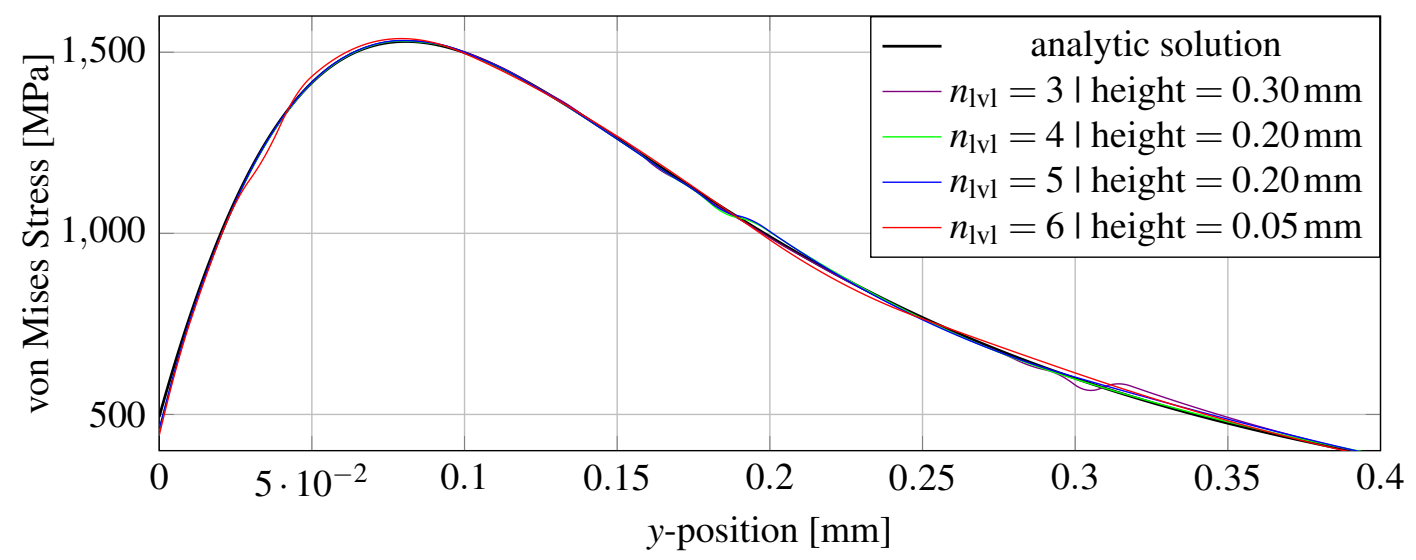

Figure 10: Maximum von Mises stresses along the symmetry axis of the locally refined models.

refined models can represent the stresses well. However, it is noticeable that a small oscillation can be seen at the $y$-position, where a switch of the hierarchy level takes place. The height of the lowest hierarchical level from Fig. 7 can be reflected in the stress analysis in Fig. 10. For comparison, a full hierarchical IGA model is tested. In order to include it in the same framework, the full IGA model is modally transformed using all modes. The oscillations still occur and thus are independent of the model reduction. In the globally refined models, which are not shown here, these small oscillations do not occur.

\section{CONCLUSIONS}

Overall, it can be concluded that it is feasible to obtain global shape functions for flexible multibody systems from hierarchically refined isogeometric finite element models. A model reduction with the Craig-Bampton method can be performed, and the global shape functions of the IGA can be smoothly included in the floating frame of reference formulation. Subsequently, an impact simulation can be performed using the penalty formulation. The penalty factor converges for hierarchically refined models, and the differences in the accuracy between the locally and globally refined models are minor. Since the locally and globally refined models are approximated with the same number of elastic coordinates and the evaluation of hierarchical NURBS in the contact routine is more complex, the overall computation time using the locally refined models is higher than the globally refined models. The computational savings achieved in the literature $[5,6,7,8]$ cannot be achieved with transient contact simulations in flexible multibody systems. However, it remains an open question whether a more efficient hierarchical NURBS algorithm can outperform a globally refined model. Adjustments to the contact algorithm are also feasible, whereby the NURBS basis of the contact body is determined in preprocessing. 


\section{REFERENCES}

[1] Schwertassek, R., Wallrapp, O.: Dynamik flexibler Mehrkörpersysteme (in German). Teubner B.G. GmbH (2014)

[2] Bathe, K.J.: Finite Element Procedures. Klaus-Jürgen Bathe, United States Watertown, MA (2014)

[3] Craig, R.R., Bampton, M.C.C.: Coupling of substructures for dynamic analyses. AIAA Journal 6(7) (jul 1968) 1313-1319

[4] Cottrell, J.A., Hughes, T.J.R., Bazilevs, Y.: Isogeometric Analysis. John Wiley \& Sons, Ltd (aug 2009)

[5] Schillinger, D., Dedè, L., Scott, M.A., Evans, J.A., Borden, M.J., Rank, E., Hughes, T.J.: An isogeometric design-through-analysis methodology based on adaptive hierarchical refinement of NURBS, immersed boundary methods, and t-spline CAD surfaces. Computer Methods in Applied Mechanics and Engineering 249-252 (dec 2012) 116-150

[6] D’Angella, D., Kollmannsberger, S., Rank, E., Reali, A.: Multi-level bézier extraction for hierarchical local refinement of isogeometric analysis. Computer Methods in Applied Mechanics and Engineering 328 (jan 2018) 147-174

[7] Noël, L., Schmidt, M., Messe, C., Evans, J., Maute, K.: Adaptive level set topology optimization using hierarchical b-splines. Structural and Multidisciplinary Optimization 62(4) (jul 2020) 1669-1699

[8] Zimmermann, C., Sauer, R.A.: Adaptive local surface refinement based on LR NURBS and its application to contact. Computational Mechanics 60(6) (aug 2017) 1011-1031

[9] Held, A., Moghadasi, A., Seifried, R.: DynManto: A matlab toolbox for the simulation and analysis of multibody systems. In: Volume 2: 16th International Conference on Multibody Systems, Nonlinear Dynamics, and Control (MSNDC), American Society of Mechanical Engineers (aug 2020)

[10] Les Piegl, W.T.: The NURBS Book. Springer-Verlag GmbH (1997)

[11] Matzen, M.E.: Isogeometrische Modellierung und Diskretisierung von Kontaktproblemen (in German). PhD thesis, University of Stuttgart, Institute of Structural Mechanics (2015)

[12] Tschigg, S.: Effiziente Kontaktberechnung in Fexiblen Mehrkörpersystemen (in German). $\mathrm{PhD}$ thesis, Hamburg University of Technology (2020)

[13] Stelzmann, U., Groth, C., Müller, G.: FEM für Praktiker 2. Strukturdynamik: Basiswissen und Arbeitsbeispiele zu FEM-Anwendungen der Strukturdynamik - Lösungen mit dem FEProgramm ANSYS (in German). Expert-Verlag (2008)

[14] Rückwald, T., Held, A., Seifried, R.: Reduced isogeometric analysis models for impact simulations. In: 17th International Conference on Multibody Systems, Nonlinear Dynamics, and Control. (2021)

[15] Matzen, M., Bischoff, M.: A weighted point-based formulation for isogeometric contact. Computer Methods in Applied Mechanics and Engineering 308 (aug 2016) 73-95

[16] Seifried, R., Hu, B., Eberhard, P.: Numerical and experimental investigation of radial impacts on a half-circular plate. Multibody System Dynamics 9(3) (2003) 265-281

[17] Johnson, K.L.: Contact Mechanics. Cambridge University Press (2004) 